\title{
Hybridizing Physical Optics with MLFMA for Efficient Scattering Computations of Three-Dimensional Complex Targets
}

\author{
Alp Manyas ${ }^{1,2}$, Özgür Ergül ${ }^{1,2}$, and Levent Gürel ${ }^{1,2}$ \\ ${ }^{1}$ Department of Electrical and Electronics Engineering \\ ${ }^{2}$ Computational Electromagnetics Research Center (BiLCEM) \\ Bilkent University, TR-06800, Bilkent, Ankara, Turkey \\ $\{$ alp,ergul,lgurel\}@ee.bilkent.edu.tr
}

\begin{abstract}
The multilevel fast multipole algorithm (MLFMA) provides accurate and efficient solutions of electromagnetic scattering problems involving large and complicated structures. On the other hand, whenever applicable, accelerations provided by approximation techniques can be useful to further improve the efficiency of solutions. In this paper, we present a hybrid technique, which combines the physical-optics (PO) method and MLFMA for efficient scattering computations of three-dimensional objects. We show that, with a careful choice of MLFMA and PO regions on the structure, the number of unknowns can be reduced and solutions can be accelerated significantly, without sacrificing the accuracy. The proposed hybrid technique is easy to implement by modifying existing MLFMA codes.
\end{abstract}

\section{INTRODUCTION}

Surface integral equations provide accurate solutions electromagnetic scattering problems involving complicated structures with metallic surfaces [1]. Simultaneous discretizations of integral equations and the scatterer lead to $N \times N$ dense matrix equations, which can be solved iteratively. For large-scale problems, matrix-vector multiplications can be performed efficiently by the multilevel fast multipole algorithm (MLFMA) in $O(N \log N)$ time using $O(N \log N)$ memory [2]. Due to its low computational complexity, MLFMA provides the solution of electromagnetics problems discretized with large numbers of unknowns on relatively inexpensive computing platforms [3],[4]. On the other hand, real-life scenarios often require multiple solutions involving different illuminations and frequencies. In those cases, accelerations provided by approximation techniques, such as the physical optics (PO), can be useful to improve the efficiency of solutions. For example, in order to reduce the number of unknowns, PO currents can be introduced on smooth and large surfaces of the object, where the PO approximation is feasible.

In this paper, we present a hybrid technique, which is based on a careful combination of PO and MLFMA for accurate and efficient scattering computations of three-dimensional complex targets. Similar to other hybrid techniques in the literature [5][12], integral equations (i.e., MLFMA) are applied only on critical regions, such as edges, tips, corners, cavities, and shadow boundaries, where the PO approximation cannot provide accurate solutions. Surface currents in those regions are solved efficiently using MLFMA, whereas the remaining currents are approximated via PO. Given a complicated object, we carefully determine the MLFMA and PO regions such that the number of unknowns is minimized, without significantly deteriorating the accuracy of results. Effectiveness of the developed implementation is demonstrated on scattering problems involving various airborne targets, such as the stealth Flamme [13].

\section{IMPLEMENTATION OF A HYBRID MLFMA-PO TECHNIQUE}

For numerical solutions of integral-equation formulations, the electric current $\boldsymbol{J}$ induced on the surface of the target is expanded in a series of basis functions, such as the RaoWilton-Glisson (RWG) functions [14], as

$$
\boldsymbol{J}(\boldsymbol{r})=\sum_{n=1}^{N} a_{n} \boldsymbol{b}_{n}(\boldsymbol{r}),
$$

where $\boldsymbol{b}_{n}$ is the $n$th basis function with a spatial support of $S_{n}$. Testing the boundary conditions on the surface using a set of testing functions $\boldsymbol{t}_{m}$ for $m=1, \ldots, N$, we obtain $N \times N$ dense matrix equations in the form of

$$
\sum_{n=1}^{N} Z_{m n} a_{n}=v_{m} \quad(m=1,2, \ldots, N) .
$$

Elements of the impedance matrix and the right-hand-side vector are derived for the electric-field integral equation (EFIE) and the magnetic-field integral equation (MFIE) as

$$
\begin{aligned}
Z_{m n}^{E} & =i k \int_{S_{m}} d \boldsymbol{r} \boldsymbol{t}_{m}(\boldsymbol{r}) \cdot \int_{S_{n}} d \boldsymbol{r}^{\prime} \boldsymbol{b}_{n}\left(\boldsymbol{r}^{\prime}\right) g\left(\boldsymbol{r}, \boldsymbol{r}^{\prime}\right) \\
& +\frac{i}{k} \int_{S_{m}} d \boldsymbol{r} \boldsymbol{t}_{m}(\boldsymbol{r}) \cdot \int_{S_{n}} d \boldsymbol{r}^{\prime} \nabla^{\prime} \cdot \boldsymbol{b}_{n}\left(\boldsymbol{r}^{\prime}\right) \nabla g\left(\boldsymbol{r}, \boldsymbol{r}^{\prime}\right) \\
v_{m}^{E} & =-\eta^{-1} \int_{S_{m}} d \boldsymbol{r} \boldsymbol{t}_{m}(\boldsymbol{r}) \cdot \boldsymbol{E}^{i n c}(\boldsymbol{r})
\end{aligned}
$$




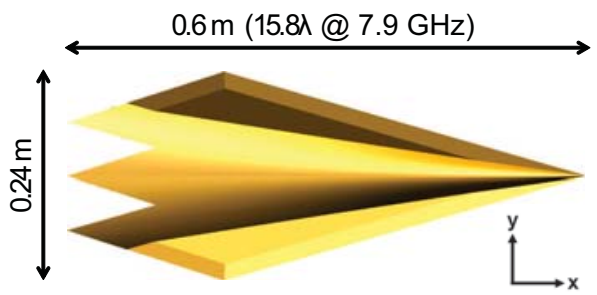

(a)

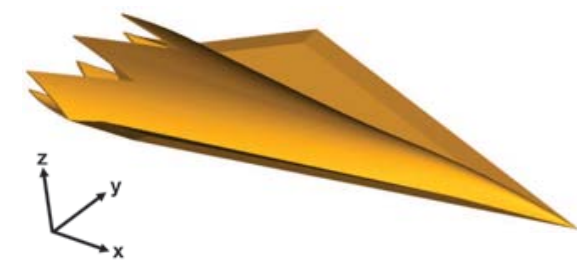

(c)

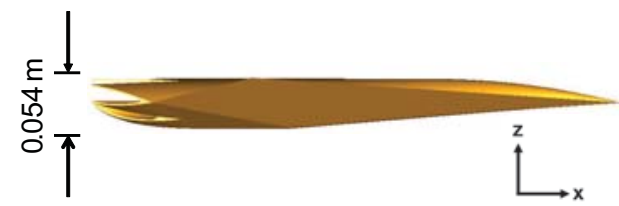

(b)

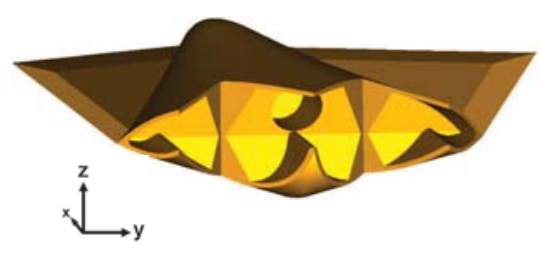

(d)

Fig. 1. Stealth airborne target Flamme; (a) top view, (b) side view, (c) isometric view, and (d) back view.

and

$$
\begin{aligned}
Z_{m n}^{M} & =\int_{S_{m}} d \boldsymbol{r} \boldsymbol{t}_{m}(\boldsymbol{r}) \cdot \hat{\boldsymbol{n}} \times \int_{P V, S_{n}} d \boldsymbol{r}^{\prime} \boldsymbol{b}_{n}\left(\boldsymbol{r}^{\prime}\right) \times \nabla^{\prime} g\left(\boldsymbol{r}, \boldsymbol{r}^{\prime}\right) \\
& -\frac{1}{2} \int_{S_{m}} d \boldsymbol{r} \boldsymbol{t}_{m}(\boldsymbol{r}) \cdot \boldsymbol{b}_{n}(\boldsymbol{r}) \\
v_{m}^{M} & =-\int_{S_{m}} d \boldsymbol{r} \boldsymbol{t}_{m}(\boldsymbol{r}) \cdot \hat{\boldsymbol{n}} \times \boldsymbol{H}^{i n c}(\boldsymbol{r}),
\end{aligned}
$$

respectively, where $P V$ indicates the principal value of the integral, $S_{m}$ is the spatial support of the $m$ th testing function, $k=\omega \sqrt{\mu \epsilon}$ is the wavenumber, and

$$
g\left(\boldsymbol{r}, \boldsymbol{r}^{\prime}\right)=\frac{\exp \left(i k\left|\boldsymbol{r}-\boldsymbol{r}^{\prime}\right|\right)}{4 \pi\left|\boldsymbol{r}-\boldsymbol{r}^{\prime}\right|}
$$

represents the homogeneous-space Green's function. In this paper, we use the combined-field integral equation (CFIE), which is the convex combination of EFIE and MFIE [15],[16], i.e.,

$$
\begin{gathered}
Z_{m n}^{C}=\alpha_{m} Z_{m n}^{E}+\left(1-\alpha_{m}\right) Z_{m n}^{M} \\
v_{m n}^{C}=\alpha_{m} v_{m}^{E}+\left(1-\alpha_{m}\right) v_{m}^{M}
\end{gathered}
$$

for $0 \leq \alpha_{m} \leq 1$.

In our hybrid MLFMA-PO technique, PO currents are initially expanded in a series of basis functions as

$$
\boldsymbol{J}_{P O}(\boldsymbol{r})=2 \hat{\boldsymbol{n}} \times \boldsymbol{H}^{i n c}(\boldsymbol{r})=\sum_{n=1}^{N_{P O}} a_{n} \boldsymbol{b}_{n}(\boldsymbol{r}),
$$

where the expansion coefficients for $n=1, \ldots, N_{P O}$ are obtained by solving a sparse matrix equation in the form of

$$
\sum_{n=1}^{N_{P O}} I_{m n} a_{n}=w_{m} \quad\left(m=1,2, \ldots, N_{P O}\right) .
$$

In (12),

$$
I_{m n}=\int_{S_{m}} d \boldsymbol{r} \boldsymbol{t}_{m}(\boldsymbol{r}) \cdot \boldsymbol{b}_{n}(\boldsymbol{r})
$$

and $w_{m}=-2 v_{m}^{M}$. After PO coefficients are determined, they are radiated to MLFMA regions via a matrix-vector multiplication, i.e.,

$$
\begin{aligned}
y_{m} & =\sum_{n=1}^{N_{P O}} Z_{m n} a_{n} \\
(m & \left.=N_{P O}+1, N_{P O}+2, \ldots, N\right),
\end{aligned}
$$

which can be performed efficiently via MLFMA. Finally, coefficients in MLFMA regions are calculated by solving the reduced matrix equation

$$
\begin{aligned}
& \sum_{n=N_{P O}+1}^{N} Z_{m n} a_{n}=v_{m}-y_{m} \\
& \quad\left(m=N_{P O}+1, N_{P O}+2, \ldots, N\right)
\end{aligned}
$$

using a Krylov-subspace algorithm accelerated with MLFMA. We note that MLFMA implementations for the matrix-vector multiplication in (14) and the matrix equation in (15) use different tree structures. Using the hybrid MLFMA-PO technique, the number of unknowns is reduced from $N$ to $\left(N-N_{P O}\right)$. The extra cost is only due to the solution of the extremely-sparse matrix equation in (12), which requires negligible time and memory. As demonstrated in the next section, the choice of MLFMA and PO regions is critical in terms of accuracy and efficiency.

\section{RESULTS}

As an example, we consider the solution of scattering problems involving the stealth airborne target Flamme depicted in Fig 1. The nose of the target is directed towards the $x$ axis, and the target is illuminated by a plane wave propagating on the $x-y$ plane at $140^{\circ}$ angle from the $x$ axis with the electric 


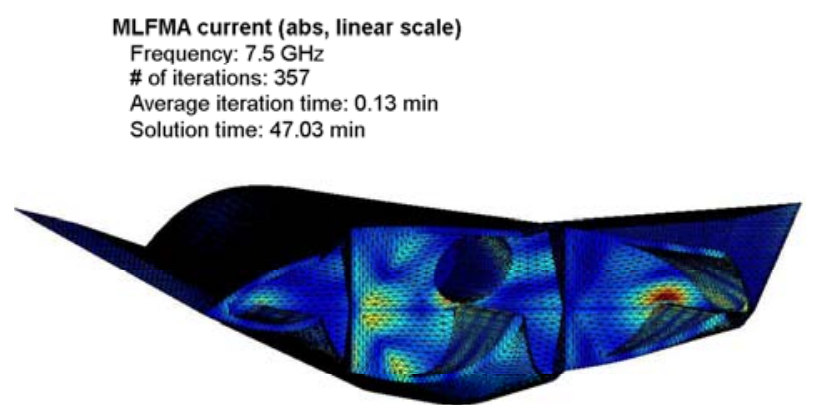

(a)

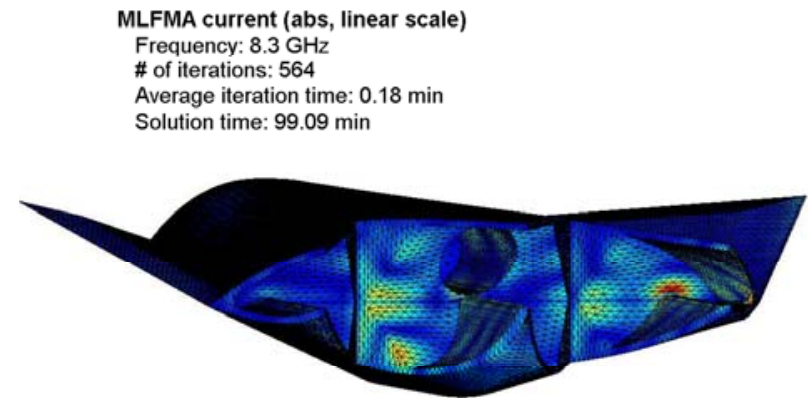

(c)

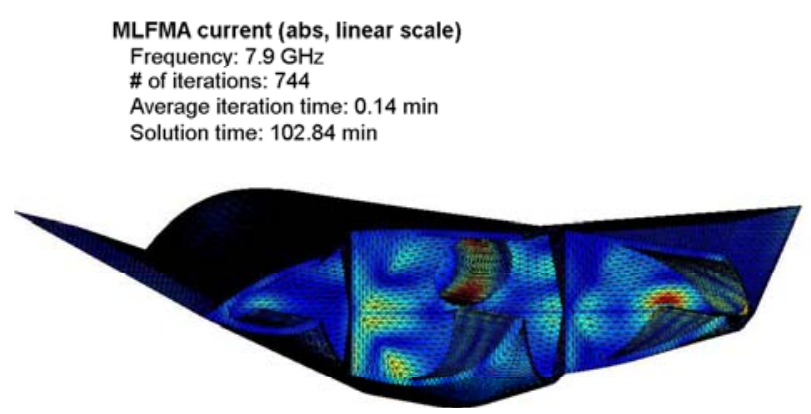

(b)

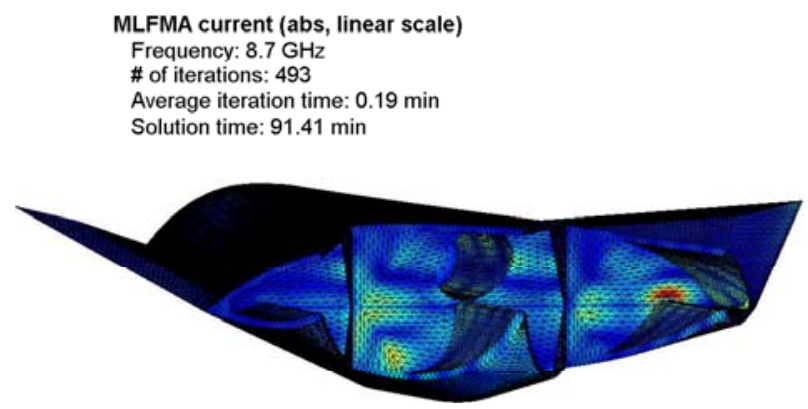

(d)

Fig. 2. Induced currents on the surface of the Flamme at (a) $7.5 \mathrm{GHz}$, (b) $7.9 \mathrm{GHz}$, (c) $8.3 \mathrm{GHz}$, and (d) $8.9 \mathrm{GHz}$ computed with MLFMA. A cavity resonance is observed inside the jet outlet at $7.9 \mathrm{GHz}$.

field polarized in the $z$ direction. Discretization of the target with $3 \mathrm{~mm}$ triangles leads to matrix equations involving 78,030 unknowns. Iterative solutions are performed by using the leastsquares QR algorithm. Fig. 2 presents the induced electric current on the surface of the Flamme at $7.5 \mathrm{GHz}, 7.9 \mathrm{GHz}$, 8.3 GHz, and 8.9 GHz, computed via MLFMA. We observe a cavity resonance inside the jet outlet at $7.9 \mathrm{GHz}$, which makes the problem difficult to handle via the PO approximation.

Fig. 3 presents the radar cross section (RCS in $\mathrm{dBms}$ ) of the Flamme at $7.9 \mathrm{GHz}$ as a function of the bistatic angle on the $x-y$ plane. In addition to MLFMA and PO solutions, RCS values are obtained by using the MLFMA-PO hybrid technique with various choices for MLFMA and PO regions. Specifically, MLFMA is employed inside the jet outlet, as well as in buffer regions around sharp edges of the target. Fig. 3(a) shows that the PO technique is significantly inaccurate and cannot provide accurate results, except for a limited range close to the forward-scattering direction. Using MLFMA in $0.1 \lambda$ buffer regions around edges (in addition to the jet outlet) reduces the average CPU time per iteration from 0.14 minutes to 0.05 minutes, corresponding to $65 \%$ reduction with respect to the full MLFMA. However, as depicted in Fig. 3(b), accuracy is not improved compared to the PO technique. When the MLFMA regions around edges are enlarged to $0.5 \lambda$, we observe a significant improvement in the accuracy of the hybrid technique, as shown in Fig. 3(c). In this case, the number of unknowns solved with MLFMA is 44,772, and the average CPU time per iteration is reduced to 0.09 minutes. Finally, as presented in Fig. 3(d), enlarging buffer regions to
$0.8 \lambda$ further improves the accuracy of the hybrid technique, but the efficiency drops due to the increasing number of unknowns solved by MLFMA.

\section{CONCLUSION}

We present a hybrid MLFMA-PO technique for efficient scattering computations of three-dimensional complex targets. The proposed technique can accelerate solutions compared to full MLFMA without sacrificing the accuracy of results, and it is easy to implement by modifying existing MLFMA codes.

\section{ACKNOWLEDGMENT}

This work was supported by the Turkish Academy of Sciences in the framework of the Young Scientist Award Program (LG/TUBA-GEBIP/2002-1-12), by the Scientific and Technical Research Council of Turkey (TUBITAK) under Research Grants 105E172 and 107E136, and by contracts from ASELSAN and SSM.

\section{REFERENCES}

[1] A. J. Poggio and E. K. Miller, "Integral equation solutions of threedimensional scattering problems," in Computer Techniques for Electromagnetics, R. Mittra, Ed. Oxford: Pergamon Press, 1973, Chap. 4.

[2] J. Song, C.-C. Lu, and W. C. Chew, "Multilevel fast multipole algorithm for electromagnetic scattering by large complex objects," IEEE Trans. Antennas Propag., vol. 45, no. 10, pp. 1488-1493, Oct. 1997.

[3] L. Gürel and Ö. Ergül, "Fast and accurate solutions of extremely large integral-equation problems discretized with tens of millions of unknowns," Electron. Lett., vol. 43, no. 9, pp. 499-500, doi:10.1049/el:20070639, Apr. 2007.

[4] Ö. Ergül and L. Gürel, "A hierarchical partitioning strategy for an efficient parallelization of the multilevel fast multipole algorithm," IEEE Trans. Antennas Propag., vol. 57, no. 6, pp. 1740-1750, Jun. 2009. 


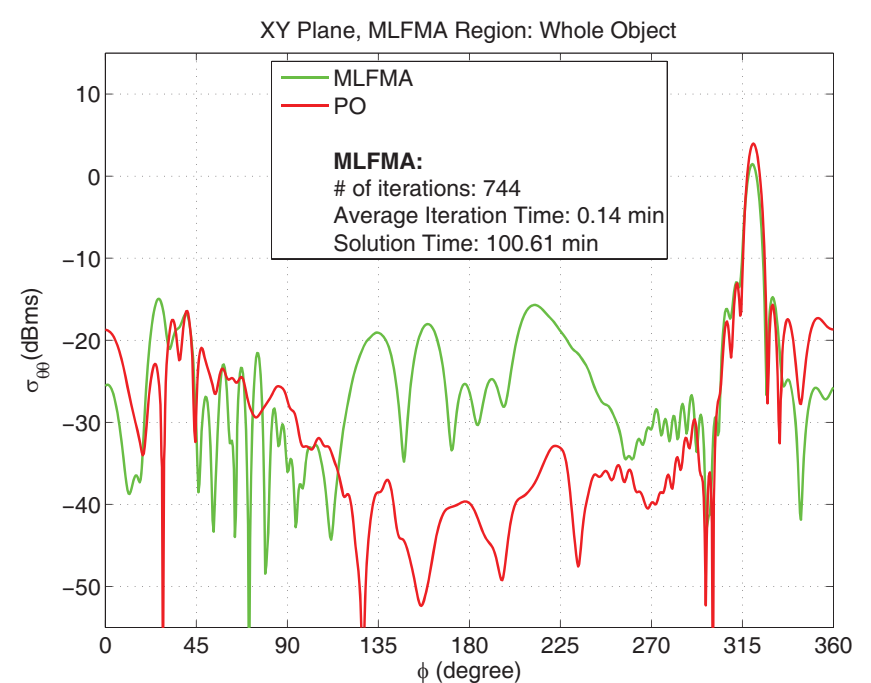

(a)

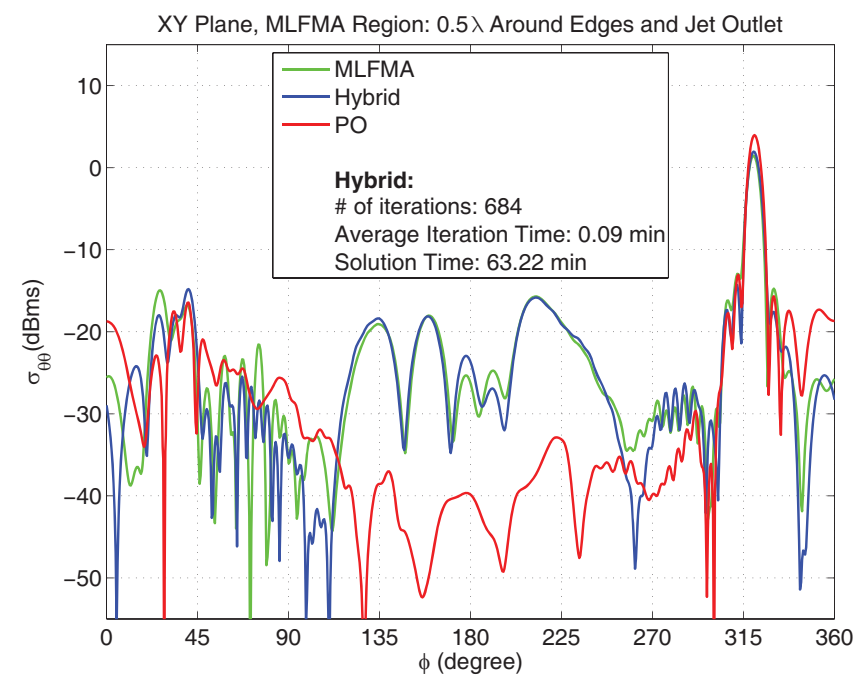

(c)

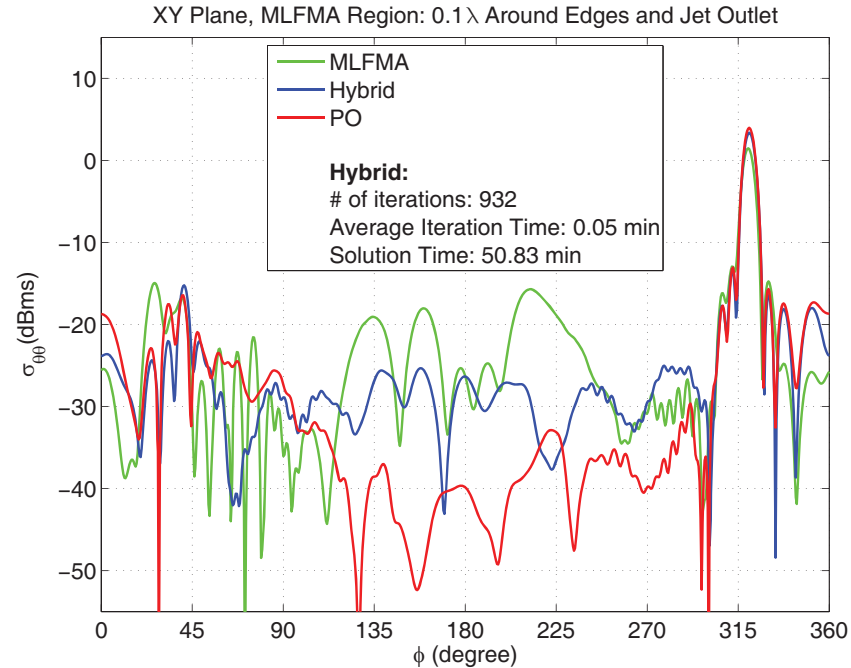

(b)

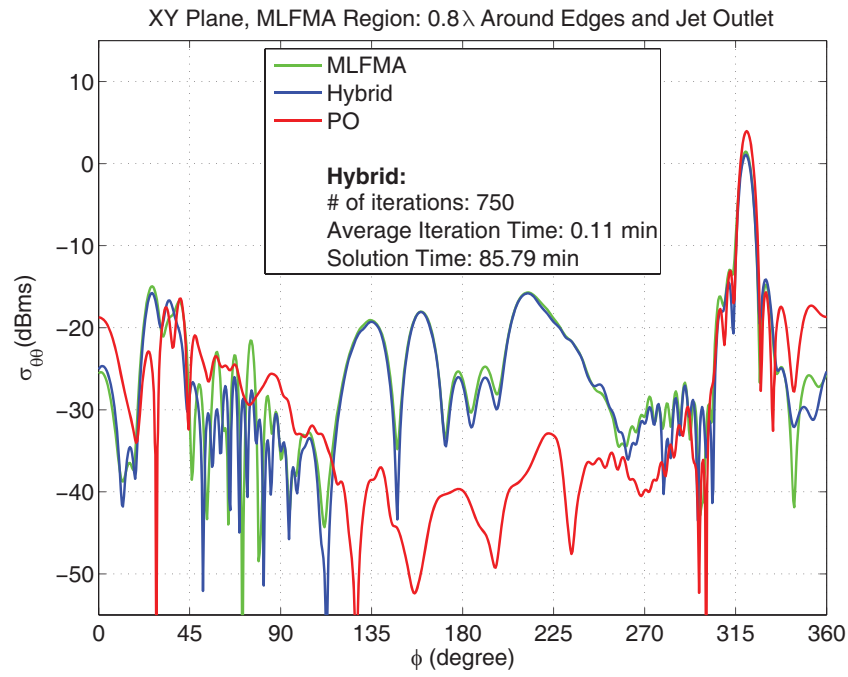

(d)

Fig. 3. Bistatic RCS of the Flamme at $7.9 \mathrm{GHz}$ obtained via PO, MLFMA, and the MLFMA-PO hybrid technique with various choices for MLFMA and PO regions.

[5] L. N. Medgyesi-Mitschang and D. S. Wang, "Hybrid solutions for scattering from perfectly conducting bodies of revolution," IEEE Trans. Antennas Propag., vol. 31, pp. 570-583, July 1983.

[6] P. K. Murthy, K. C. Hill, and G. A. Thiele, "A hybrid-iterative method for scattering problems," IEEE Trans. Antennas Propag., vol. 34, pp. 1173 1180, Oct. 1986

[7] C. S. Kim and Y. Rahmat-Samii, "Low profile antenna study using the physical optics hybrid method (POHM)," Proc. IEEE Int. Symp. Antennas and Propagat. Soc. Meet., London, 1991.

[8] D. B. Bouche, F. A. Molinet, and R. Mittra, "Asymptotic and hybrid techniques for electromagnetic scattering," Proc. IEEE, vol. 81, no. 12, pp. 1658-1684, Dec. 1993.

[9] U. Jakobus and F. M. Landstorfer, "Improvement of the PO-MoM hybrid method by accounting for effects of perfectly conducting wedges," IEEE Trans. Antennas Propag., vol. 43, no. 10, pp. 1123-1129, Oct 1995.

[10] R. E. Hodges and Y. Rahmat-Samii, "An iterative current-based hybrid method for complex structures," IEEE Trans. Antennas Propag. vol. 45, no. 2, pp. 265-276, Feb. 1997.

[11] M. Djordjevic and B. M. Notaros, "Higher order hybrid method of moments-physical optics modeling technique for radiation and scattering from large perfectly conducting surfaces," IEEE Trans. Antennas
Propag., pp. 800-813, vol. 53, no. 2, Feb. 2005.

[12] A. Tzoulis and T. F. Eibert, "A hybrid FEBI-MLFMM-UTD method for numerical solutions of electromagnetic problems including arbitrarily shaped and electrically large objects," IEEE Trans. Antennas Propag., pp. 3358-3366, vol. 53, no. 10, Oct. 2005.

[13] L. Gürel, H. Bağc1, J. C. Castelli, A. Cheraly, and F. Tardivel, "Validation through comparison: measurement and calculation of the bistatic radar cross section (BRCS) of a stealth target," Radio Sci., vol. 38, no. 3, Jun. 2003.

[14] S. M. Rao, D. R. Wilton, and A. W. Glisson, "Electromagnetic scattering by surfaces of arbitrary shape," IEEE Trans. Antennas Propag., vol. 30 , no. 3, pp. 409-418, May 1982.

[15] J. R. Mautz and R. F. Harrington, "H-field, E-field, and combined field solutions for conducting bodies of revolution," $A E \ddot{U}$, vol. 32 , no. 4 , pp. 157-164, Apr. 1978

[16] Ö. Ergül and L. Gürel, "Iterative solutions of hybrid integral equations for coexisting open and closed surfaces," IEEE Trans. Antennas Propag., vol. 57, no. 6, pp. 1751-1758, Jun. 2009. 